\title{
TAREBIA GRANIFERA (LAMARCK, 1822) EN LA REGIÓN HIDROLÓGICA GRIJALVA-USUMACINTA EN TABASCO, MÉXICO
}

\author{
Luis José Rangel Ruiz, Jaquelina Gamboa Aguilar, Miguelina García \\ Morales, Óscar Manuel OrTiz LeZama
}

Laboratorio de Malacología, División Académica de Ciencias Biológicas, Universidad Juárez Autónoma de Tabasco. Carretera Villahermosa-Cárdenas Km 0.5 entronque con Saloya. C. P. 94250 Villahermosa, Tabasco, MÉXICO. Tel. y Fax (993) 354-43-08. ljrangel@msn.com

\section{Rangel Ruiz, L. J., J. Gamboa Aguilar, M. García Morales \& O. M. Ortiz Lezama. 2011. Tarebia granifera (Lamarck, 1822) en la región hidrológica Grijalva-Usumacinta en Tabasco, México. Acta Zool. Mex. (n. s.), 27(1): 103-114.}

RESUMEN. Después del primer hallazgo de Tarebia granifera en septiembre de 2007 en la laguna Tintal, en la Reserva de la Biósfera Pantanos de Centla (RBPC), se realizó una búsqueda en 40 lagunas, 6 ríos, un arroyo y dos drenes: 14 sitios en el municipio de Centla, 3 en Centro, 11 en Paraíso, 1 en Nacajuca, 3 en Cárdenas, 14 en Macuspana y 3 en Jonuta. Los muestreos se realizaron en mayo y septiembre de 2008 y mayo y septiembre de 2009. El muestreo fue al azar tomándose 10 muestras con una draga Van Veen de cinco litros. De los 49 sitios, sólo en 11 de tres municipios se recolectó T. granifera: en el municipio de Centro en L. Pucte y L. la Ceiba; Centla en L. Guanal, L. San Isidro, L. Viento, L. Larga, L. Tintal y L. Concepción; y Macuspana en L. Loncho, L. Ismate-Chilapilla y L. Chilapa. El polígono de distribución de T. granifera está entre las coordenadas UTM 509588-590058 X y 1989183-1976233 Y

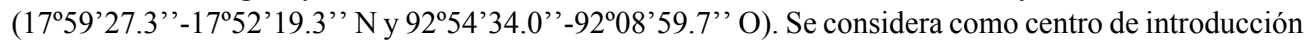
y dispersión a la Ciudad de Villahermosa. Las localidades que presentaron las máximas abundancias fueron: L. Tintal con 7,944 individuos, L. Pucte con 7,048 y L. Ismate Chilapilla con 1,268. Se comprueba la competencia de esta especie con Melanoides tuberculata siendo favorecida T. granifera.

Palabras clave: Tarebia granifera, Tabasco, distribución y abundancia.

Rangel Ruiz, L. J., J. Gamboa Aguilar, M. García Morales \& O. M. Ortiz Lezama. 2011. Tarebia granifera (LAMARCK, 1822) in hydrologic region Grijalva-Usumacinta in Tabasco, Mexico. Acta Zool. Mex. (n. s.), 27(1): 103-114.

ABSTRACT. After the first finding of Tarebia granifera in September 2007 in Lake Tintal in the Reserva de la Biosfera Pantanos de Centla, a search was conducted in 40 lakes, 6 rivers, 1 stream and two drains: 14 sites from the municipality of Centla, 3 from Centro, 11 from Paraiso, 1 from Nacajuca, 3 from Cardenas, 14 from Jonuta and 3 from Macuspana. Sampling was conducted in September 2008 and May and September 2009. The sampling was random taking 10 samples using five liters Van Veen grab. Tarebia granifera was collected in 11 sites out of 49 in three municipalities: in the town of Centro is

Recibido: 23/04/2010; aceptado: 13/09/2010. 
was found in L. Pucte and L. La Ceiba; Centla in L. Guanal, L. San Isidro, L. Viento, L. Larga, L. Tintal and L. Concepcion; and Macuspana in L. Loncho, L. Ismate-Chilapilla and L. Chilapa. The polygon of distribution of T. granifera is between the coordinates UTM 509588-590058 X and 1989183-1976233 Y

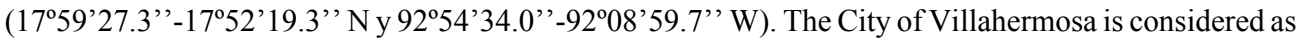
an introduction and dispersal center. The localities that presented the highest abundance values were: $\mathrm{L}$. Tintal with a total 7,944 individuals, L. Pucte with 7,048 and L. Ismate-Chilapilla with 1,268. This study confirms the competence between $T$. granifera and Melanoides tuberculata being the former favored. Key words: Tarebia granifera, Tabasco, distribution and abundance.

\section{INTRODUCCIÓN}

La región Hidrológica Grijalva-Usumacinta es considerada como una región prioritaria por las autoridades ambientales de México y por la comunidad científica internacional, por su enorme biodiversidad, alta productividad, funciones ecológicas y servicios ambientales que presta (CONABIO 1997, Toledo 2003). Por lo anterior, es de suma importancia darle seguimiento a la introducción de especies exóticas invasoras, ya que son consideradas la segunda causa de pérdida de biodiversidad a nivel global después de la pérdida de hábitat (Vitousek et al. 1996, Leung et al. 2002). Las especies invasoras desplazan a las especies nativas de flora y fauna por competencia directa, depredación, transmisión de enfermedades, modificaciones del hábitat, alteración de la estructura de los niveles tróficos y sus condiciones biofísicas (CONABIO 2006). Hasta el 2007 sólo se tenían registradas para México dos especies de moluscos invasores de alto impacto para la biodiversidad, Melanoides tuberculata (Müller 1774) y Corvicula spp ya presentes en nuestro país (Mifsut \& Martínez 2007).

El primer registro de $T$. granifera en la República Mexicana se presentó para el Lago de Catemaco al sur del estado de Veracruz (Naranjo-García et al. 2005) y el segundo en las cabeceras de los ríos Tuxpan y Tecolutla en el mismo estado (TapiaVega \& López-López 2008).

Para el estado de Tabasco la única especie de gasterópodo registrada como introducida era M. tuberculata para el Río Grijalva, cerca de la ciudad de Villahermosa (Contreras-Arquieta 1995), para el "Parque Estatal la Sierra" (Rangel-Ruiz y Gamboa-Aguilar 2001), y para la Reserva de la Biosfera Pantanos de Centla (RBPC) (Cruz-Ascencio et al. 2003, Albarran-Melze 2009).

Es importante la frecuente asociación entre $T$. granifera y $M$. tuberculata especies que tienen el mismo hábitat, que explotan los mismos recursos y quienes establecen una competencia entre sus poblaciones, resultando la mayoría de las veces favorecida T. granifera (Naranjo-García et al. 2005). Esta especie es altamente competitiva, puede llegar incluso a desplazar especies endémicas de los sitios en donde ha sido introducida, y además es un hospedero intermediario potencial de parásitos del hombre y peces de importancia comercial (Pointier et al. 1998).

En mayo de 2008, producto de una colecta en la laguna Tintal localizada en el municipio de Centla se recolectaron 1264 organismos de $T$. granifera, lo cual motivó 
a realizar una búsqueda de esta especie en 40 lagunas, 6 ríos, un arroyo y dos drenes: 14 sitios en el municipio de Centla, tres en Centro, 11 en Paraíso, uno en Nacajuca, tres en Cárdenas, 14 en Macuspana y tres en Jonuta. El objetivo principal de este trabajo es establecer la presencia y distribución actual de $T$. granifera para el estado de Tabasco y conocer su asociación con M. tuberculata.

\section{ÁREA DE ESTUDIO}

Los sitios de colecta se ubican dentro de la Región Hidrológica Grijalva-Usumacinta la cual se localiza en el sureste de la República Mexicana y administrativamente comprende los estados de Tabasco, Chiapas y pequeñas porciones de Campeche. El área de estudio se localiza en la provincia de la Llanura Costera del Golfo Sur, en la subprovincia Llanura y Pantanos Tabasqueños (Toledo 2003).

Las localidades por municipio son las siguientes:

Municipio de Cárdenas:

Río Santana, en la desembocadura a la Laguna Machona y Río Santana Ranchería Santuario Primera Sección.

Municipio de Centla:

L. San Pedrito, L. Punteada, L. Guanal, L. San Isidro, L. Coco, L. Cometa, L. Viento, L. Concepción, L. Larga, L. Ídolos, L. Tintal, L. Chichicastle, L. Santa Anita y L. Provecho.

Municipio de Centro:

L. Pucte, L. Ceiba y L. Ismate-Chilapilla.

Municipio de Jonuta

Dren Narváez Norte, Dren Narváez Sur y L. Narváez. Municipio de Macuspana:

L. Landeros, L. Sargazal, L. Loncho, L. Sauzo, L. San Isidro el Jobo, L. Cantemoc, Arroyo San Cristóbal Ranchería Gustavo A. Madero, Río Chilapa, L. Chilapa, L. El Bayo, L. Limón, L. Francisco Bates, L. San Sebastián y L. Pajaral. Municipio de Paraíso:

L. Mecoacán en cinco sitios (Boca Negra, Boca Grande, Boca Limón, Hormiguero, Los Ángeles y Arrastradero), Río Cuxcuchapa (cercano al campo Mecoacán), L. Negrita, L. Las Flores, L. Puente Ostión, L. Redonda y Río Cuxcuchapa (Ranchería Buena Ventura).

Municipio de Nacajuca:

El Río el Mango.

\section{MATERIALES Y MÉTODOS}

Se realizaron cuatro muestreos, en mayo y septiembre de 2008 y 2009. En cada laguna la elección de los sitios de muestreo fue al azar y se tomaron 10 muestras en cada 
sitio con ayuda de una draga Van Veen de cinco litros, la cual se lanzó el número de veces necesario para obtener el volumen de una cubeta de cinco litros siendo esta la unidad de medida. Cada muestra se tamizó in situ a través de un juego de tamices de $0.5,2.0$ y $10.0 \mathrm{~mm}$ de abertura de luz. Para los ríos, el muestreo se realizó sobre el margen en un tramo de $100 \mathrm{~m}$ lineales, utilizando una red de golpeo, raspando los márgenes desde una profundidad de $1 \mathrm{~m}$ hasta la superficie. Los organismos fueron recolectados, almacenados en bolsas de plástico, rotulados y fijados en alcohol al $70 \%$ para su posterior cuantificación en laboratorio.

Para establecer la existencia de una posible competencia de las poblaciones de $T$. granifera y $M$. tuberculata en las localidades con mayor frecuencia y abundancia relativa, se calculo la distribución de frecuencias de las dos especies, durante el periodo de estudio (Pointier et al. 1998). Además para establecer el grado de asociación que existe entre las abundancias de las dos especies por localidad se realizo un análisis de correlación.

\section{RESULTADOS}

De los 49 sitios de muestreo sólo en once lagunas de tres municipios se recolectó a T. granifera: en el municipio de Centro en L. Pucte y L. La Ceiba; en el municipio de Centla en L. Guanal, L. San Isidro, L. Viento, L. Larga, L. Tintal y L. Concepción y en el municipio de Macuspana en L. Loncho, L. Ismate-Chilapilla y L. Chilapa. El polígono de distribución de T. granifera se encuentra entre las coordenadas UTM 509588-590058 X y 1989183-1976233 Y (17²59'27.3'”-1752'19.3'” N y 92 $54^{\circ} 34.0^{\prime}$ ' $-92^{\circ} 08^{\prime} 59.7^{\prime}$ ' O). (Fig. 1, Cuadro 1). Todas las localidades se encuentran incluidas en la cuenca Río Grijalva-Villahermosa (Subcuencas Río Chilapilla y Río Grijalva) y la cuenca Río Usumacinta (Subcuencas R. Usumacinta y R. Chilapa) (Cuadro 1).

El primer registro de $T$. granifera se obtuvo en la L. Tintal en mayo de 2008 para septiembre de ese mismo año en L. Concepción, L. Ismate Chilapilla y L. Pucte. Para mayo de 2009 en L. Guanal, L. Loncho, L. San Isidro, L. Viento y L. Ceiba y recientemente en septiembre de 2009 en L. Larga y L. Chilapa (Cuadro 2).

Las localidades que presentaron las máximas abundancias fueron: L. Tintal con una abundancia total de 7,944 individuos, L. Pucte con 7,048 y L. Ismate-Chilapilla con 1,268. El comportamiento de la abundancia de $T$. granifera en L. Tintal y L. Ismate Chilapilla es errático, y en L. Pucte se ha incrementado de forma paulatina en los tres muestreos realizados. En los ocho sitios restantes no fue posible determinar un comportamiento ya que son de reciente invasión (Cuadro 2).

Considerando la Ciudad de Villahermosa como centro de introducción y dispersión de $T$. granifera por la conectividad por ríos a las lagunas invadidas, las probables rutas de dispersión se presentan en el Cuadro 3. 


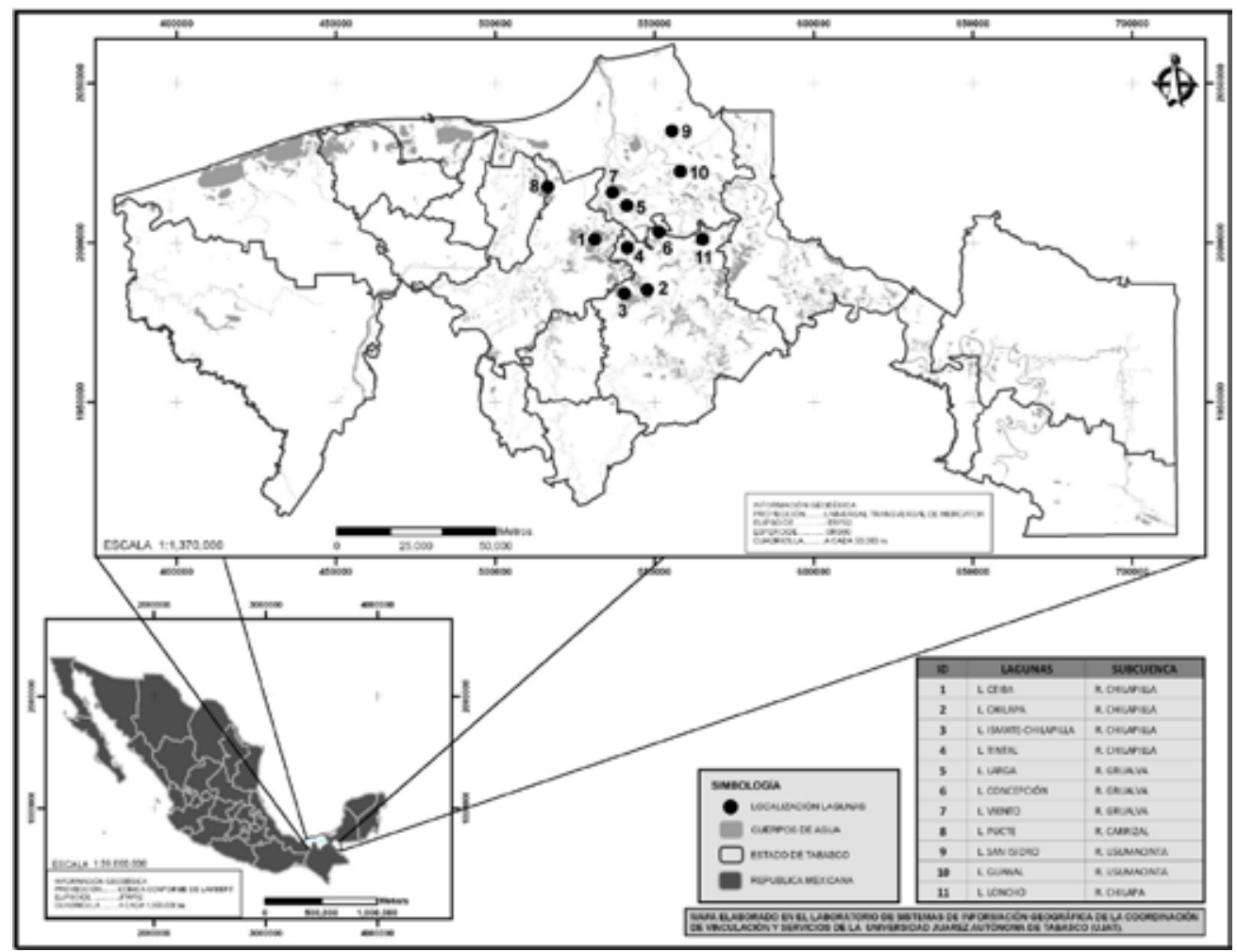

Figura 1. Área de distribución de Tarebia granifera en el estado de Tabasco.

Para establecer la posible competencia de las poblaciones de $T$. granifera y $M$. tuberculata se consideraron sólo las localidades con mayor frecuencia y abundancia relativa: en L. Tintal las abundancias de $T$. granifera siempre fueron superiores a las de $M$. tuberculata y se observa una disminución de la población de M. tuberculata en mayo de 2008 para mantenerse en una proporción similar a este valor durante los dos muestreos posteriores; L. Pucte es el único sitio hasta el momento en donde se observa un desplazamiento claro de $M$. tuberculata, ya que su abundancia relativa ha disminuido de septiembre 2008 a septiembre 2009, obteniéndose un coeficiente de correlación de $-0.9861(p \leq 0.05)$ que indica una asociación negativa; y en L. IsmateChilapilla la abundancia de T. granifera disminuye de septiembre 2008 a mayo 2009 para nuevamente incrementar su abundancia en septiembre 2009 (Fig. 2).

\section{DISCUSIÓN}

En el estado de Tabasco actualmente se tienen registradas varias especies de organismos acuáticos invasores; los peces Oreochromis aureus (Steindachner 1864), $O$. 
Cuadro 1. Distribución de Tarebia granifera en once lagunas del estado de Tabasco 2008-2009.

\begin{tabular}{lcccccc}
\hline & \multicolumn{2}{c}{ Coordenadas } & & & \\
\cline { 2 - 3 } \multicolumn{1}{c}{ Lagunas } & X & Y & & Cuenca & Subcuenca & Municipio \\
\hline Ceiba & 531307 & 2001049 & R. Grijalva-Villahermosa & R. Chilapilla & Centro \\
Chilapa & 547729 & 1985296 & R. Grijalva-Villahermosa & R. Chilapilla & Macuspana \\
Ismate- & 540589 & 1984012 & R. Grijalva-Villahermosa & R. Chilapilla & Macuspana \\
Chilapilla & & & & & \\
Tintal & 567436 & 1997471 & R. Grijalva-Villahermosa & R. Chilapilla & Centla \\
Larga & 541419 & 2011703 & R. Grijalva-Villahermosa & R. Grijalva & Centla \\
Concepción & 451485 & 2003396 & R. Grijalva-Villahermosa & R. Grijalva & Centla \\
Viento & 536857 & 2015756 & R. Grijalva-Villahermosa & R. Grijalva & Centla \\
Pucte & 516547 & 2017532 & R. Grijalva-Villahermosa & R. Carrizal & Centro \\
San Isidro & 555517 & 2035123 & R. Usumacinta & R. Usumacinta & Centla \\
Guanal & 558223 & 2022356 & R. Usumacinta & R. Usumacinta & Centla \\
Loncho & 565111 & 2001112 & R. Usumacinta & R. Chilapa & Macuspana \\
\hline
\end{tabular}

UTM (Universal Transverse Mercator Projection).

Cuadro 2. Abundancia de Tarebia granifera y Melanoides tuberculata en el estado de Tabasco.

\begin{tabular}{|c|c|c|c|c|c|c|c|c|}
\hline \multirow[b]{2}{*}{ Localidad } & \multicolumn{2}{|c|}{ May 2008} & \multicolumn{2}{|c|}{ Sep 2008} & \multicolumn{2}{|c|}{ May 2009} & \multicolumn{2}{|c|}{ Sep 2009} \\
\hline & $T . g$ & M.t & $T . g$ & M.t & T.g & M.t & $T . g$ & M.t \\
\hline L. Guanal & & & & & 34 & 1886 & 26 & 509 \\
\hline L. Concepción & & & 1 & 333 & 9 & 92 & & \\
\hline L. Loncho & & & & & 375 & 12 & 4 & 6 \\
\hline L. San Isidro & & & & & 3 & 3250 & 44 & 2713 \\
\hline L. Viento & & & & & 159 & 161 & 424 & 76 \\
\hline L. Larga & & & & & & & 1 & 65 \\
\hline L. Tintal & 1264 & 689 & 3214 & 830 & 2525 & 886 & 941 & 292 \\
\hline L. Ismate-Chilapilla & & & 426 & 287 & 304 & 59 & 538 & 1778 \\
\hline L. Chilapa & & & & & & & 226 & 810 \\
\hline L. La Ceiba & & & & & 13 & 4 & 1 & 61 \\
\hline L. Pucte & & & 1407 & 1777 & 2648 & 1168 & 2993 & 1138 \\
\hline
\end{tabular}

May: Mayo, Sep: Septiembre, T.g : T. granifera, M.t : M. Tuberculata.

mossambicus (Peters 1852) y Tilapia rendalli (Boulenger 1897) de origen africano, Ctenopharyngodon idella (Valenciennes 1844) de origen asiático y Parachromis managüensis (Günther 1867) y Parachromis motaguensis (Günther 1867) nativos de Nicaragua en América Central y más recientemente el "plecostomus o pez diablo" 
Cuadro 3. Probables rutas de dispersión de Tarebia granifera por ríos a partir de la Ciudad de Villahermosa en el estado de Tabasco.

\begin{tabular}{lcl}
\hline \multicolumn{1}{c}{ Laguna } & $\mathrm{km}$ & Ruta de dispersión por ríos de Tabasco \\
\hline L. Guanal & 62.23 & Grijalva-Usumacinta \\
L. Concepción & 42.73 & $\begin{array}{l}\text { Grijalva-Jahuacte-Hormiguero-Los Negros-Tasajera-Paquillal-Los } \\
\text { Naranjos }\end{array}$ \\
L. Loncho & 67.06 & Grijalva-Bitzal-Hormiguero-Los Negros-Tasajera \\
L. San Isidro & 66.41 & Grijalva-Usumacinta \\
L. Viento & 40.00 & Grijalva-Hormiguero \\
L. Larga & 38.47 & Grijalva-Hormiguero \\
L. Tintal & 34.22 & Grijalva-Jahuacte-Hormiguero \\
L. Ismate-Chilapilla & 29.27 & Grijalva-Jahuacte-Bernardino-Chilapilla \\
L. Chilapa & 39.36 & Grijalva-Jahuacte-Bernardino-Chilapilla \\
L. La Ceiba & 26.55 & Grijalva-Jahuacte \\
L. Pucte & 28.61 & Grijalva-Carrizal-Río González \\
\hline
\end{tabular}

$\mathrm{km}=$ Distancia en kilómetros de la Cd. de Villahermosa a cada laguna invadida por $T$. granifera en línea recta .

Pterygoplichthys pardalis (Casteinau 1855) (Mendoza 2007, Espinosa-Pérez y Daza-Zepeda 2005); y el caracol Melanoides tuberculata (Cruz-Ascencio et al. 2003, Albarran-Melze et al. 2009). Por lo anterior y tomando en cuenta que la Región Hidrológica Grijalva-Usumacinta es considerada una región prioritaria, resulta urgente el desarrollo de programas de prevención que impidan la introducción de nuevas especies, y de erradicación y/o control y manejo de las especies ya introducidas (Mifsut \& Martínez 2007).

Tarebia granifera es una especie originaria del sureste de Asia e introducida accidentalmente al continente Americano en Texas alrededor de 1935 y en Florida entre 1940 y 1947 (Murray \& Woopschall 1965, Abbot 1952). A partir de esta fecha se han presentado introducciones a varias islas de las Antillas menores, países de Centroamérica y Sudamérica.

Si bien Tarebia granifera actualmente presenta una distribución amplia en las Islas del Caribe (Puerto Rico, República Dominicana, Haití, Santa Lucia, Granada, Antigua, Cuba, Jamaica y Martinica), Sudamérica (Venezuela) y en los estados del sur de Estados Unidos (Texas y Florida, Hawai), (Pointier et al. 1994, 1998, Hyslop 2002), en México Tarebia granifera se había registrado exclusivamente en el estado Veracruz (Naranjo et al. 2005, Tapia-Vega \& Oliveros-Jiménez 2008), por lo cual este trabajo representa el primer registro de $T$. granifera para el estado de Tabasco ampliando su rango de distribución con once lagunas cercanas a la ciudad de Villahermosa.

La forma de introducción es desconocida, sin embargo se atribuye al acuarismo, ya que especies de caracoles como físidos, planórbidos, pomáceos y tiáridos entre 
TINTAL

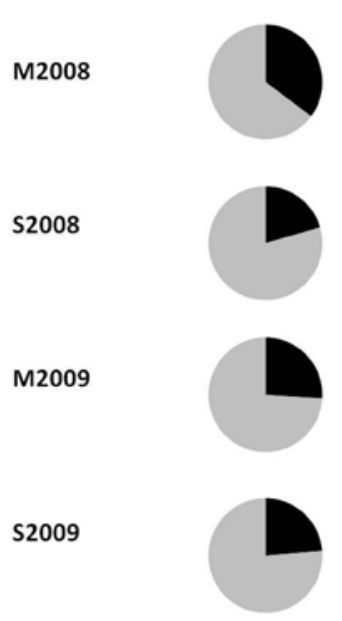

PUCTE
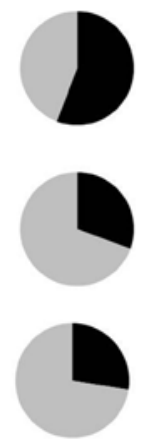
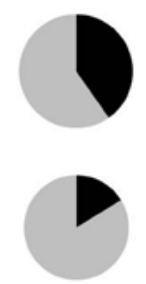

ISMATE CHILAPILLA

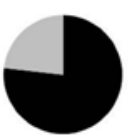

Figura 2. Distribución de frecuencia de la abundancia de Tarebia granifera (gris) y Melanoides tuberculata (negro) en tres localidades del estado de Tabasco (mayo 2008-septiembre 2009).

$$
\mathrm{M}=\text { mayo, } \mathrm{S}=\text { septiembre. }
$$

otras especies frecuentemente se encuentran entre las plantas acuáticas que se venden en acuarios de la ciudad. Estos se pueden encontrar en diferentes estados de desarrollo en forma de masas de huevos, juveniles o adultos. Al lavar los acuarios estos caracoles son arrojados de forma voluntaria o involuntaria junto con el agua de desecho, contaminando los sistemas acuáticos, lo que aunado a la resistencia de estos caracoles a soportar condiciones adversas logran sobrevivir y establecerse con éxito en nuevos cuerpos de agua lénticos o lóticos (Contreras Arquieta et al. 1995, Naranjo-García et al. 2005, Letelier et al. 2007, Lachner et al. 1970).

La propuesta de que la Ciudad de Villahermosa fue el centro de introducción de T. granifera y que la ruta probable de dispersión fue el Río Grijalva, está basada en dos aspectos: a) en los centros urbanos cercanos a las lagunas con presencia actual de esta especie no existe actividad de acuarismo como la que se desarrolla en la capital del estado; b) se considera que el centro del área de distribución de una especie tiende a presentarse en las poblaciones con altas densidades (abundancias) (Rapoport \& Monjeau 2003). Por esto último podemos señalar que L. Tintal, L. Pucte y L. IsmateChilapilla fueron las primeras localidades en ser colonizadas por $T$. granifera y que actualmente fungen como las aéreas de dispersión para el resto del estado de Tabasco.

Las fechas de introducción a las lagunas que pertenecen a la RBPC se presentaron pocos meses antes de su hallazgo, lo cual se puede afirmar debido a que en la reserva se realiza un monitoreo de comunidades acuáticas de moluscos durante las épocas 
de estiaje (mayo) y lluvias (septiembre), a partir de septiembre de 2003. Se puede asegurar entonces que para la L. Tintal esta introducción fue anterior al mes de mayo de 2008; para L. Concepción a septiembre del mismo año; para L. Guanal, L. Loncho y L. Viento a mayo de 2009; y para L. Larga a septiembre de 2009. Para las lagunas fuera de la RBPC como L. Ismate-Chilapilla y L. Pucte no se conoce con exactitud cuándo fue la introducción, ya que en estas lagunas el monitoreo comenzó a partir de septiembre de 2008. Sin embargo si se tiene la certeza que para L. La Ceiba fue previa al mes de mayo de 2009 y para L. Chilapa a septiembre del mismo año.

La velocidad de dispersión de T. granifera en el estado fue favorecida por la inundación a finales del 2007 (octubre y noviembre), que afectó alrededor del 80\% de la superficie del estado de Tabasco (Centro de Satélites de Información en Situaciones de Crisis (ZKI) del DLR 2007). Actualmente la dispersión de T. granifera se lleva a cabo por el movimiento periódico del lirio acuático por ríos o canales durante la época de lluvias, con dirección en general del sur al norte, este comportamiento se ha observado desde el 2003 que comenzó el programa de monitoreo de moluscos en la Reserva de la Biosfera Pantanos de Centla. Otros probables métodos de dispersión pueden ser debidos al movimiento de las aves trasportando entre sus plumas o patas algunos caracoles o al transporte de lanchas y barcazas que transitan por estos sistemas fluviales (Naranjo-García et al. 2005).

Los valores de abundancia de Tarebia granifera registrados hasta el momento muestran una enorme variación y por lo tanto una inestabilidad en sus poblaciones, debido principalmente a los diferentes tiempos de introducción y al establecimiento de competencia con otras especies de moluscos en particular con M. tuberculata (Pointier et al. 1998, 1994).

Las tres localidades donde suponemos se establecieron los primeros caracoles y presentaron la mayor abundancia promedio fueron L. Pucte $(2,349.33 \pm 834.12)$, L. Tintal $(1,986.00 \pm 1,066.43)$ y L. Ismate-Chilapilla $(422.67 \pm 117.04)$ las cuales muestran valores superiores a los registrados en el estado de Veracruz en el Río Tecolutla y Río Tuxpan recolectados en agosto y septiembre de 2007 con 122 y 249 individuos respectivamente (en $10 \mathrm{~m}^{2}$ ) (Tapia-Vega \& Oliveros-Jiménez 2008); los recolectados en las mismas localidades en diciembre de 2007 con 485 y 264 ind (colecta manual y tamizado) (Oliveros-Jiménez et al. 2008) y en cinco ríos de Venezuela en donde la densidad varió de 23 a 90 ind por metro cuadrado (Pointier et al. 1994). Este comportamiento confirma que los sistemas lénticos albergan una mayor abundancia de $T$. granifera que en los sistemas lóticos, debido principalmente a la falta de un sustrato adecuado (vegetación a orillas del rio o arroyo) y a la velocidad de la corriente que actúan como limitantes al establecimiento de las poblaciones de caracoles (Pointier et al. 1994).

En la mayoría de los muestreos cuando se presentaron las dos especies de tiáridos, la abundancia relativa de $T$. granifera fue mayor que $M$. tuberculata, lo que demuestra 
que es un mejor competidor a pesar de que estas dos especies comparten las mismas estrategias reproductivas, como son un alto potencial biótico por ser especies ovovivíparas y partenogenéticas y desarrollo rápido y madurez precoz (Pereda et al. 1990). A pesar de observarse una disminución de la abundancia relativa de $M$. tuberculata hasta el momento no se ha observado un fenómeno de exclusión o extinción, este comportamiento ha sido registrado con anterioridad en Venezuela, Cuba y Martinica. Además estas dos especies han demostrado su eficiencia como agentes de control biológico sobre hospederos intermediarios de parásitos de importancia médica en los mismos países anteriormente mencionados (Perera et al. 1990, Pointier et al, 1994).

Tarebia granifera al igual que $M$. tuberculata son hospederos intermediarios de parásitos de humanos en China como son Clonorchis sinensis (Looss 1907) (gusano o duela del hígado) y Paragonimus westermani (Kerbert 1878) (gusano o duela del pulmón), sin embargo Michelson (1992) la considera susceptible a la infección de miracidios de $P$. westermani sin alcanzar el grado de huésped intermediario. Afortunadamente estas especies de tremátodos no han sido registradas todavía en el Continente Americano. Lo que sí está confirmado es que son hospederos de Centrocestus formosanus (Nishigori 1924) el cual infecta peces saturando sus branquias y asfixiándolos, por lo que causa grandes pérdidas económicas a las pesquerías y acuacultura en varios países de América (Karatayev et al. 2009, Aguirre-Muñoz et al. 2009, Mendoza 2004). En México C. formosanus ha sido registrado parasitando 59 especies de peces nativos e introducidos en 12 estados del centro y sureste de la República Mexicana (Pérez-Ponce de León et al. 2007), incluyendo Tabasco en las localidades de: La Granja Piscícola de Teapa, El Espino, Yumká, Río Puyacatengo y El Rosario (Salgado-Maldonado et al. 2005). Scholz \& Salgado-Maldonado (2000) señalan que por la distribución y efectos sobre las poblaciones de peces en agua continentales de C. formosanus en México el pronóstico es grave.

AGRADECIMIENTOS. A PEMEX Exploración y Producción Región Sur por haber financiado el desarrollo del proyecto Evaluación de la Hidrodinámica y Comunidades Bióticas en la Reserva de la Biosfera Pantanos de Centla, y del Programa de Monitoreo Hidrológico y de calidad del agua del Sistema Ambiental Regional de Tabasco, del cual forma parte esta investigación.

\section{LITERATURA CITADA}

Abbot, R. T. 1952. A study of an intermediate snail host (Thiara granifera) of the oriental lung fluke (Paragonimus). Proceeding of the United States National Museum, 102: 71-116.

Aguirre-Muñoz, A., R. Mendoza-Alfaro, H. Arredondo, L. Arriaga, E. Campos, S. Contreras-Balderas $\dagger$, M. E. Gutiérrez, F. J. Espinosa, I. Fernández, L. Galaviz, F. J. García, D. Lazcano, M. Martínez, M. E. Meave, R. A. Medellín, E. Naranjo, M. T. Olivera, M. Pérez, G. Rodríguez, G. Salgado, A. Samaniego, E. Suárez, H. Vibrans, J. A. Zertuche. 2009. Especies exóticas invasoras: impactos sobre las poblaciones de flora y fauna, los procesos ecológicos y la economía, pp. 277-318. In: Carabias, J., J. Soberón \& R. Dirzo (Eds.). Capital natural de México, vol. II: Estado de conservación y tendencias de cambio. CONABIO, México, 809 pp. 
Albarran-Melze, N. C., L. J. Rangel-Ruiz \& J. Gamboa Aguilar. 2009. Distribución y abundancia de Melanoides tuberculata (Gastropoda: Thiaridae) en la Reserva de la Biosfera Pantanos de Centla, Tabasco, México. Acta Zoológica Mexicana (n. s.), 25(1): 93-104.

CONABIO. 1997. Regiones prioritarias para la conservación de México. www.conabio.gob.mx/dtos/ rpcm.

CONABIO. Aridamérica, GECI, TNC. 2006. Especies invasoras de alto impacto a la biodiversidad: Prioridades en México. Ciudad de México, Mayo, 42 p.

Contreras-Arquieta, A., G. Guajardo-Martínez \& S. Contreras-Balderas. 1995. Redescripción del caracol exógeno Thiara (Melanoides) tuberculata (Müller, 1774) (Gastropoda: Thiaridae) y su distribución en México. Publicaciones Biológicas - F.C.B./U.A.N.L., México, 8: 1-16.

Cruz-Ascencio, M., R. Florido, A. Contreras-Arquieta \& A. J. Sánchez. 2003. Registro del caracol exótico Thiara (Melanoides) tuberculata (Müller, 1774) (Gastropoda: Thiaridae) en la Reserva de la Biosfera Pantanos de Centla. Universidad y Ciencia, 19: 101-103.

Espinosa-Pérez, H. \& A. Daza-Zepeda. 2005. PECES, Cap. 10:225-240. In: Bueno, J., F. Álvarez y S. Santiago (Eds.) Biodiversidad del estado de Tabasco, 386 p. Instituto de Biología, UNAM-CONABIO, México, $370 \mathrm{p}$.

Hyslop, E. J. 2002. Additions to the freshwater malacofauna of Jamaica. Revista de Biología Tropical, 262-263.

Karatayev, A. Y., L. E. Burlakova, A. A. Karatayev \& D. K. Padilla. 2009. Introduction, distribution, spread, and impacts of exotic freshwater gastropods in Texas. Hydrobiologia, 619: 181-194.

Lachner, E. A., C. R. Robins \& W. R. Courtenay. 1970. Exotic fishes and other aquatic organisms introduced into North America. Smithsonian Contributions to Zoology, 59: 1-29.

Letelier, V. S., A. M. L. Ramos \& L. G. M. Huaquín. 2007. Moluscos dulceacuícolas exóticos de Chile. Revista Mexicana de Biodiversidad, 78: 9-13.

Leung, B., D. M. Lodge, D. Finnoff, J. F. Shogren, M. A. Lewis \& G. Lamberti. 2002. An ounce of prevention or a pound of cure: Bioeconomic risk analysis of invasive species. Proceeding of the Royal Society Biological Sciences, 269: 2407-2413.

March, M. I. J. \& M. J. Martínez. 2007. Especies invasoras de alto impacto a la biodiversidad. Prioridades en México. IMTA-CONABIO-GECI-AridAmérica-The Nature Conservancy, 72 pp.

Mendoza, R. 2004. Introduction of invasive aquatic species on the inland waters ecosystems of Mexico, in: L. A. Meyerson, K. A. Ciruna \& E. Watson (eds.). The ecological and socio-economic impacts of invasive alien species on island ecosystems: Report of an expert's consultation. Global invasive Species Programme, Washington, D. C.

Mendoza, R., S. Contreras, C. Ramírez, P. Koleff, P. Álvarez \& V. Aguilar. 2007. Los peces diablo: especies invasoras de alto impacto. Biodiversitas, 70: 1-5.

Michelson, E. H. 1992. Thiara granifera: a victim of authoritarianism. Malacological Review, 25: 67-71.

Mifsut, M. I. J. \& Martinez J. M. 2007. Especies invasoras de alto impacto a la biodiversidad. Prioridades en México. IMTA-CONABIO-GECI-AridAmérica-The Nature Conservancy, 72 pp.

Murray, H. D. \& L. J. Woopschall. 1965. Ecology of Melanoides tuberculata (Müller) and Tarebia granifera (Lamarck) in South Texas. American Malacological Union Incompany, 25-25.

Naranjo-García, E., M. E. Diupotex-Chong \& G. R. Familiar. 2005. Tarebia granifera (Lamarck, 1822) (Gastropoda: Prosobranchia: Pachychilidae) en el Lago de Catemaco, Veracruz, México. VI Congreso Latinoamericano de Malacología. Instituto Smithsonian de Investigaciones Tropicales, 101.

Oliveros-Jiménez, J. E., P. Tapia-Vega \& E. López-López. 2008. Análisis morfométrico y comportamiento poblacional de Tarebia granifera presente en los ríos Tuxpan y Tecolutla, Veracruz. En: 
Sánchez A. J., Hidalgo M. M. G. Arriaga W. S. L. \& Contreras S. W. M. Perspectivas en Zoología Mexicana, 31-42.

Perera, G., M. Yong, J. R. Ferrer, C. Arrinda \& O. Amador. 1990. Effectiveness of three biological control agents against intermediate host of snail-mediated parasites in Cuba. Malacological Review, 23: 47-52.

Pérez-Ponce de León, G., L. García-Prieto \& B. Mendoza-Garfias. 2007. Trematode parasites (Platyhelminthes) of wildlife vertebrates in Mexico. Zootaxa, 1543: 1-247.

Pointier, J. P. 2001. El control biológico de los moluscos vectores intermediarios de los esquistosomas: el ejemplo de la región Caribe. Vitae: Academia Biomédica Digital. Ed.: CAIBCO-Centro de Análisis de Imágenes Biomédicas Computarizadas. Sitio: http://caibco.ucv.ve/caibco/vitae/VitaeOcho/ Articulos/MedicinaTropical/ArchivosPDF/MedicinaTropical.PDF Junio/Agosto número 8.

Pointier, J. P., R. N. Incani, C. Balzan, P. Chrosciechowski \& S. Prypchan. 1994. Invasion of the rivers of the Littoral Central Region of Venezuela by Thiara granifera and Melanoides tuberculata (Mollusca: Prosobranchia: Thiaridae) and the absence of Biomphalaria glabrata, snail host of Schistosoma mansoni. The Nautilus, 17: 124-128.

Pointier, J. P., S. Samadi, P. Jarne \& B. Delay. 1998. Introduction and spread of Thiara granifera (Lamarck, 1822) in Martinique, French West Indies. Biodiversity and Conservation, 7: 1277-1290.

Rangel, R. L. J. y J. A. Gamboa. 2001. Diversidad malacológica en la Región Maya. I. "Parque Estatal de la Sierra", Tabasco, México. Acta Zoológica Mexicana (n.s.), 82: 1-12.

Rapoport, E. H. \& J. A. Monjeau. 2003. Areografía, 22-30. In: Morrone J.J. \& J.LL. Bousquets (Eds.) Una perspectiva latinoamericana de la biogeografía. Facultad de Ciencias UNAM, México, $277 \mathrm{p}$.

Salgado-Maldonado, G., R. Pineda-López, L. García-Magaña, S. López-Jiménez, V. M. VidalMartínez \& L. Aguirre-Macedo. 2005. Helmintos parásitos de peces dulceacuícolas, Cap 6:145166. In: Bueno, J., F. Álvarez \& S. Santiago (Eds.) Biodiversidad del estado de Tabasco. Instituto de Biología, UNAM-CONABIO, México, 386 p.

Sholz, T. \& G. Salgado-Maldonado. 2000. The introduction and dispersal of Centrocestus formosanus (Nishigori, 1924) (Digenea: Heterophyidae) in Mexico: A review. American Midland Naturalist, 143: 185-200.

Tapia-Vega, P. \& J. E. Oliveros-Jiménez. 2008. Estructura poblacional de Tarebia granifera (Lamarck, 1822) (Gastropoda: Prosobranchia: Thiaridae) en la parte alta de los ríos Tuxpan y Tecolutla, Veracruz. En: Sánchez A. J., Hidalgo M. M. G. Arriaga W. S. L. \& Contreras S. W. M. Perspectivas en Zoología Mexicana, 43-53.

Toledo, A. 2003. Ríos, costas, mares. Hacia un análisis integrado de las regiones hidrológicas de México. SEMANARP-INE, Colegio de México, $114 \mathrm{p}$.

Vitousek, P. M., C. M. D’Antonio, LL. Loope \& R. Westbrooks. 1996. Biological invasions as global environmental change. American Scientist, 84: 468-478.

Centro de Satélites de Información en Situaciones de Crisis. 2007. (http://www.zki.caf.dlr.de/applications/2007/mexico/142_en.html. 